PROCEEDINGS OF THE

AMERICAN MATHEMATICAL SOCIETY

Volume 134, Number 8, Pages 2473-2478

S 0002-9939(06)08304-3

Article electronically published on February 8, 2006

\title{
UNIVERSAL ABSOLUTE EXTENSORS IN EXTENSION THEORY
}

\author{
ALEX KARASEV AND VESKO VALOV
}

(Communicated by Alexander N. Dranishnikov)

\begin{abstract}
Let $L$ be a countable and locally finite CW complex. Suppose that the class of all metrizable compacta of extension dimension $\leq[L]$ contains a universal element which is an absolute extensor in dimension $[L]$. Our main result shows that $L$ is quasi-finite.
\end{abstract}

\section{INTRODUCTION}

In this note we deal with one of the central problems in extension theory that can be described as follows. Consider a CW complex $L$. Suppose that the class of all metrizable compacta of extension dimension $\leq[L]$ has a universal element which is an absolute extensor in dimension $[L]$. What can be said about the properties of the complex $L$ ? It is known [4, Theorem 2.5] that the situation described above occurs when $L$ is a finite complex. The main purpose of this note is to show that such a complex $L$ must be necessarily quasi-finite. We do not know whether this condition is also sufficient. Note that quasi-finite $\mathrm{CW}$ complexes were introduced in [7] as complexes which provide the solution of the following problem: characterize all complexes $P$ such that there exists a $P$-invertible map of a metrizable compactum of extension dimension $\leq[P]$ onto the Hilbert cube. Note also that existence of such a map for a complex $P$ implies the existence of a universal metrizable compactum of extension dimension $[P]$. Consequently, if $L$ is quasi-finite, it guarantees the existence of a universal compactum of extension dimension $[L]$.

As an application of our result we show that there is no universal compactum of the same cohomological dimension which is an absolute extensor with respect to spaces of given cohomological dimension. A similar result [9] was known only for the case of integral cohomological dimension.

\section{Preliminaries}

In this and all subsequent sections "complex" means "a countable and locally finite CW complex". All spaces under consideration are assumed to be Tychonov and all maps are continuous. The letter " $L$ " will be reserved to denote a complex. In this note, for spaces $X$ and $Y$, the notation $Y \in A E(X)$ will always mean that

Received by the editors June 8, 2004 and, in revised form, March 14, 2005.

2000 Mathematics Subject Classification. Primary 55M10; Secondary 54F45.

Key words and phrases. Absolute extensors, universal compacta, extension dimension, cohomological dimension, quasi-finite complexes.

The authors were partially supported by their NSERC grants.

(C)2006 American Mathematical Society Reverts to public domain 28 years from publication 2473 
every map $f: A \rightarrow Y$, defined on a closed subspace $A$ of $X$, admits an extension over $X$. By $[L]$ we denote the extension type of a complex $L$ and by $e-\operatorname{dim} X$ we denote the extension dimension of a space $X$ [5, 6]. For a normal space $X$, the inequality $e-\operatorname{dim} X \leq[L]$ means that $L \in A E(X)$. More information about extension dimension and extension types can be found in 2, 4.

We say that a map $f: X \rightarrow Y$ is $[L]$-soft $[4$ if for each Polish space $Z$ with $e-\operatorname{dim} Z \leq[L]$, for each closed subspace $A$ of $Z$, and for any two maps $g: Z \rightarrow Y$ and $h: A \rightarrow X$ such that $f \circ h=\left.g\right|_{A}$, there exists a map $\bar{h}: Z \rightarrow X$ extending $h$ and satisfying the condition $f \circ \bar{h}=g$.

Let $\mathcal{B}$ be a certain class of spaces. We shall say that a space $X$ is an absolute extensor in dimension $[L]$ for the class $\mathcal{B}$ (notation $X \in A E([L], \mathcal{B})$ ) if $X \in A E(Y)$ for every $Y$ from $\mathcal{B}$ such that $e-\operatorname{dim} Y \leq[L]$. We shall denote the class of all metrizable compacta by $\mathcal{C}$ and the class of all Polish spaces by $\mathcal{P}$. The following remark is trivial.

Proposition 2.1. Let $f: X \rightarrow Y$ be an $[L]$-soft map. Then $X \in A E([L], \mathcal{P})$ iff $Y \in A E([L], \mathcal{P})$.

Let $X$ be a normal space. A pair of spaces $V \subset U$ is called $X$-connected if for every closed subspace $A \subset X$ any map of $A$ to $V$ can be extended to a map of $X$ into $U$. Suppose that $\mathcal{B}$ is a certain subclass of the class of normal spaces. A pair of spaces $V \subset U$ is called $[L]$-connected with respect to $\mathcal{B}$ if for every space $X \in \mathcal{B}$ with $e-\operatorname{dim} X \leq[L]$ the pair $V \subset U$ is $X$-connected. In what follows we will need the following observation from [1, Proposition A.1] (recall that in this note we consider only countable complexes).

Proposition 2.2. Let $L$ be a complex and let $V \subset U$ be a pair of Polish spaces. If this pair is $[L]$-connected with respect to Polish spaces, then it is $[L]$-connected with respect to all normal spaces.

We say [7 that a complex $L$ is quasi-finite if for every finite subcomplex $P$ of $L$ there exists a finite subcomplex $P^{\prime}$ of $L$ containing $P$ such that the pair $P \subset P^{\prime}$ is $[L]$-connected with respect to Polish spaces.

The following theorem provides a characterization of quasi-finite complexes. Note that equivalences from (a) through (e) were obtained by Chigogidze in [3, Theorem 2.1] and the equivalence of these properties to (f) follows from [7, Theorem 3.1].

Theorem 2.3. Let $L$ be a complex. Then the following statements are equivalent:

(a) $e-\operatorname{dim} \beta X \leq[L]$ whenever $X$ is a space with $e-\operatorname{dim} X \leq[L]$.

(b) $e-\operatorname{dim} \beta X \leq[L]$ whenever $X$ is a normal space with $e-\operatorname{dim} X \leq[L]$.

(c) $e-\operatorname{dim} \beta\left(\bigoplus\left\{X_{t} \mid t \in T\right\}\right) \leq[L]$ whenever $T$ is an arbitrary indexing set and $X_{t}, t \in T$, is a separable metrizable space with $e-\operatorname{dim} X_{t} \leq[L]$.

(d) $e-\operatorname{dim} \beta\left(\bigoplus\left\{X_{t} \mid t \in T\right\}\right) \leq[L]$ whenever $T$ is an arbitrary indexing set and $X_{t}, t \in T$, is a Polish space with $e-\operatorname{dim} X_{t} \leq[L]$.

(e) There exists an $[L]$-invertible map $f: X \rightarrow I^{\omega}$ where $X$ is a metrizable compactum with $e-\operatorname{dim} X \leq[L]$.

(f) $L$ is quasi-finite. 


\section{RESULTS}

Let $\mathcal{B}$ be a subclass of the class of normal spaces. We say that a complex $L$ possesses the connected pairs property with respect to $\mathcal{B}$ if for any metrizable compactum $K$ with $e-\operatorname{dim} K \leq[L]$ there exists a metrizable compactum $C$ containing $K$ such that $e-\operatorname{dim} C \leq[L]$ and the pair $K \subset C$ is $[L]$-connected with respect to $\mathcal{B}$.

Lemma 3.1. Let $T$ be an arbitrary indexing set and let $\left\{X_{t} \mid t \in T\right\}$ be a collection of Polish spaces such that $e-\operatorname{dim} X_{t} \leq[L]$ for each $t \in T$. Let $X=\bigoplus\left\{X_{t} \mid t \in T\right\}$. Suppose that $K \subset C$ is a pair of metrizable compacta such that $e-\operatorname{dim} K \leq[L]$. If the pair $K \subset C$ is $[L]$-connected with respect to Polish spaces, then this pair is $\beta X$-connected.

Proof. Let $A$ be a closed subset of $\beta X$ and let $f: A \rightarrow K$ be a map. Consider the adjunction space $Y=X \cup_{f} f(A)$. Note that $Y$ can be viewed as the disjoint union of two subspaces, homeomorphic to $(X-A)$ and $f(A)$, respectively. We claim that $e-\operatorname{dim} Y \leq[L]$. Indeed, $f(A)$ is a closed subspace of $K$, and therefore $e-\operatorname{dim} f(A) \leq[L]$. Further, $X-A$ is an open subset of $X$. Note that $X$ is metrizable and therefore perfectly normal. Therefore, the claim follows from the countable sum theorem. Observe also that Proposition 2.2 allows us to assume that the pair $K \subset C$ is $L$-connected with respect to normal spaces. Hence, the identity map $i$ of a copy of $f(A)$ in $Y$ to a copy of $f(A)$ in $K$ can be extended to a map $j: Y \rightarrow C$. Let $p: X \cup A \rightarrow Y=X \cup_{f} f(A)$ be the natural projection and let $\bar{f}=j \circ p$. Then $\bar{f}: X \cup A \rightarrow C$ extends $f$ to $X \cup A$. Now the unique extension $\beta \bar{f}$ of $\bar{f}$ over $\beta X$ yields the required extension of $f$.

Lemma 3.2. Let $L$ be a complex possessing the connected pairs property with respect to Polish spaces and let $X$ be a compactum. Suppose that each pair $K \subset C$ of metrizable compacta with $e-\operatorname{dim} C \leq[L]$ is $X$-connected provided $K \subset C$ is $[L]$-connected with respect to Polish spaces. Then for every metrizable space $Y \in A E([L], \mathcal{C})$ with $e-\operatorname{dim} Y \leq[L]$ we have $Y \in A E(X)$

Proof. Let $A$ be a closed subset of $X$ and let $f: A \rightarrow Y$ be a map. Note that $f(A)$ is a metrizable compactum and $e-\operatorname{dim} f(A) \leq[L]$. Therefore there exists a metrizable compactum $B$ with $e-\operatorname{dim} B \leq[L]$ such that the pair $f(A) \subset B$ is $[L]$-connected with respect to Polish spaces. Hence, by our hypotheses, the pair $f(A) \subset B$ is $X$-connected. Since $f$ can be viewed as a map sending $A$ to a copy of $f(A)$ inside $B$, this map can be extended to a map $f^{\prime}: X \rightarrow B$. Since $Y \in A E([L], \mathcal{C})$, the homeomorphism identifying a copy of $f(A)$ in $B$ with a copy of $f(A)$ in $Y$ can be extended to a map $h: B \rightarrow Y$. Clearly the map $\bar{f}=h \circ f^{\prime}: X \rightarrow Y$ is an extension of $f$.

Everywhere below by $\operatorname{cov}(X)$ we denote the set of all open covers of a space $X$. If $A$ is a subset of $X$ and $\omega \in \operatorname{cov}(X)$ we denote the star of $A$ with respect to $\omega$ by $\operatorname{St}(A, \omega)$. We say that $\nu \in \operatorname{cov}(X)$ is a strong star-refinement of if for each $V \in \nu$ there exists $W \in \omega$ such that $S t(V, \nu) \subset W$. The following set of notations is borrowed from [1. For a cover $\Sigma \in \operatorname{cov}(X)$ we denote by $\Sigma^{(k)}$ its " $k$-dimensional skeleton", i.e. the set of all points in $X$ at which order of $\Sigma$ is at most $k+1$. Thus we let $\Sigma^{(k)}=\left\{x \in X \mid \operatorname{ord}_{\Sigma} x \leq k+1\right\}$. For elements $s_{0}, s_{1}, \ldots, s_{n} \in \Sigma$ with 
non-empty intersection $\bigcap_{i=0}^{n} s_{i}$ we define a "closed $n$-dimensional simplex"

$$
\left[s_{0}, s_{1}, \ldots, s_{n}\right]=\bigcup_{i=0}^{n} s_{i} \backslash \bigcup\left\{s \in \Sigma \mid s \neq s_{i}, i=0,1, \ldots, n\right\}
$$

and its "interior" $\left\langle s_{0}, s_{1}, \ldots, s_{n}\right\rangle=\bigcap_{i=0}^{n} s_{i} \cap \Sigma^{(n)}$. It is easy to check that the $n$-skeleton consists of $n$-simplices

$$
\Sigma^{(n)}=\bigcup\left\{\left[s_{i_{0}}, s_{i_{1}}, \ldots, s_{i_{n}}\right] \mid \bigcap_{k=0}^{n} s_{i_{k}} \neq \varnothing\right\}
$$

and that any "simplex" consists of its "boundary" and its "interior"

$$
\left[s_{0}, s_{1}, \ldots, s_{n}\right]=\bigcup_{m=0}^{n}\left[s_{0}, \ldots, \widehat{s}_{m}, \ldots, s_{n}\right] \cup\left\langle s_{0}, s_{1}, \ldots, s_{n}\right\rangle .
$$

Clearly, $\Sigma^{(k)}$ is closed in $X$ and $\Sigma^{(n)}=X$ if the cover $\Sigma$ has order $n+1$. Note also that the "interiors" of distinct $k$-dimensional "simplices" are mutually disjoint and

$$
\Sigma^{(k)}=\bigcup\left\{\left\langle s_{i_{0}}, s_{i_{1}}, \ldots, s_{i_{n}}\right\rangle \mid \bigcap_{k=0}^{n} s_{i_{k}} \neq \varnothing\right\} \cup \Sigma^{(k-1)} .
$$

The following lemma can be interpreted as a "weak" version of Lemma 3.10 from [8].

Lemma 3.3. Let $X$ be a compactum and let $Z$ be a paracompact space such that any compact subspace of $Z$ is finitely dimensional in the sense of usual Lebesgue dimension. Let $g: Y \rightarrow Z$ be a surjection with the following property: for every $z \in Z$ and its neighborhood $U(z)$ in $Z$ there exists a smaller neighborhood $V(z)$ of $z$ such that $g^{-1}(V(z)) \in A E(X)$. Then for any $\omega \in \operatorname{cov}(Z)$ and for any map $f: X \rightarrow Z$ there exists a map $\tilde{f}: X \rightarrow Y$ such that the maps $f$ and $g \circ \tilde{f}$ are $\omega$-close.

Proof. Note that $f(X) \subset Z$ is compact and therefore $\operatorname{dim} f(X)=n<\infty$ for some $n$. We let $\omega_{0}=\omega$ and inductively construct a sequence of covers $\omega_{1}, \omega_{2}, \ldots, \omega_{n}$ as follows. Suppose $\omega_{i} \in \operatorname{cov}(Z)$ is already constructed and let $\nu$ be a strong starrefinement of $\omega_{i}$. For each $z \in Z$ we choose $U(z) \in \nu$ containing $z$ and find a smaller neighborhood $V(z) \subset U(z)$ of $z$ having the property

$$
g^{-1}(V(z)) \in A E(X)
$$

We let $\omega_{i+1}=\{V(z) \mid z \in Z\}$. Obviously, $\omega_{i+1}$ is a strong star-refinement of $\omega_{i}$.

Let $\Sigma \in \operatorname{cov}(f(X))$ be a finite strong star-refinement of $\omega_{n}$ restricted on $f(X)$ such that $\operatorname{ord} \Sigma \leq n+1$. We put $\widehat{\Sigma}=\left\{f^{-1}(U) \mid U \in \Sigma\right\}$. Clearly $\widehat{\Sigma}$ is a finite open cover of $X$ of order $\leq n+1$. By induction we construct a sequence of maps $f_{0}, f_{1}, \ldots, f_{n}$ such that $f_{k}: \widehat{\Sigma}^{(k)} \rightarrow Y$ with the property

$$
g\left(f_{k}(x)\right) \in \operatorname{St}\left(f(x), \omega_{n-k}\right)
$$

for all $k$. In order to construct $f_{0}$ for each element $s \in \widehat{\Sigma}$ we choose a point $P_{s} \in g^{-1}(f(s))$, and then for every "closed one-dimensional simplex" $[s]$ we let $\left.f_{0}\right|_{[s]}=P_{s}$. Suppose that $f_{k}$ has already been constructed. It suffices to define $f_{k+1}$ on the "interior" $\langle\sigma\rangle$ of each "simplex" $[\sigma]=\left[s_{0}, s_{1}, \ldots, s_{k+1}\right]$. Since $\widehat{\Sigma}$ is finite and the "interiors" of "closed $k$-dimensional simplices" are mutually disjoint, we can consider each simplex independently. Let $[\sigma]^{\prime}=[\sigma] \cap \widehat{\Sigma}^{(k)}$. Since $\Sigma$ is a strong star-refinement of $\omega_{n}$ (and consequently of $\omega_{n-k}$ ) and because of property 
(*), we can find $W_{\sigma} \in \omega_{n-k}$ such that $g\left(f_{k}\left([\sigma]^{\prime}\right)\right) \subset S t\left(W_{\sigma}, \omega_{n-k}\right)$. Since $\omega_{n-k}$ is a strong star-refinement of $\omega_{n-k-1}$ and by the construction of $\omega_{n-k-1}$, there exists an element $V_{\sigma} \in \omega_{n-k-1}$ possessing property $(\dagger)$ and such that $S t\left(W_{\sigma}, \omega_{n-k}\right) \subset V_{\sigma}$. Since $g^{-1}\left(V_{\sigma}\right) \in A E(X)$ we can extend the map $\left.f_{k}\right|_{[\sigma]^{\prime}}$ to a map $f_{k+1}:[\sigma] \rightarrow$ $g^{-1}\left(V_{\sigma}\right) \subset Y$. It is easy to check that property $(*)$ is satisfied for $f_{k+1}$.

Finally, we let $\widetilde{f}=f_{n}$.

The following theorem provides a characterization of quasi-finite complexes in terms of connected pairs property.

Theorem 3.4. A complex $L$ possesses the connected pairs property with respect to Polish spaces iff $L$ is quasi-finite.

Proof. The "if" part follows from [8, Proposition 2.4]. In order to establish the "only if" part we shall show that $L$ satisfies property (d) from Theorem 2.3. Let $\left\{X_{t} \mid t \in T\right\}$ be a collection of Polish spaces where $T$ is an arbitrary indexing set and assume that $e-\operatorname{dim} X_{t} \leq[L]$ for each $t \in T$. Let $X=\bigoplus\left\{X_{t} \mid t \in T\right\}$. We need to show that $e-\operatorname{dim} \beta X \leq[L]$. Let $A$ be a closed subset of $\beta X$ and let $f: A \rightarrow L$ be a map. Consider an $[L]$-soft map $g: Y \rightarrow L$, where $Y$ is a Polish space with $e-\operatorname{dim} Y \leq[L]$. The existence of such a map follows from [2, Proposition 5.9].

We claim that the map $g$ satisfies conditions of Lemma 3.3. Indeed, consider $z \in L$ and its open neighborhood $U(z)$. Then $U(z)$ contains a neighborhood $V(z)$ of $z$ in $L$ which is an absolute extensor. Proposition 2.1 implies that $g^{-1}(V(z)) \in A E([L], \mathcal{P})$. Subsequently applying Lemma 3.1 and Lemma 3.2 (for the pair $\left.g^{-1}(V(z)) \subset g^{-1}(V(z))\right)$, we conclude that $g^{-1}(V(z)) \in A E(\beta X)$. This proves the claim. Note also that the same arguments show that $Y \in A E(\beta X)$.

Since $L$ is $A N R$-space, there exists an open cover $\omega \in \operatorname{cov}(L)$ such that any two $\omega$-close maps to $L$ are homotopic. Applying Lemma 3.3 to the maps $g: Y \rightarrow L$, $f: A \rightarrow L$, and to the cover $\omega$, we obtain a map $\tilde{f}: A \rightarrow Y$ such that $f$ and $g \circ \tilde{f}$ are $\omega$-close. Since $Y \in A E(\beta X)$, we can extend $\tilde{f}$ to a map $\bar{f}: \beta X \rightarrow Y$. Let $f^{\prime}=g \circ \bar{f}: \beta X \rightarrow L$. Note that $\left.f^{\prime}\right|_{A}$ is $\omega$-close to $f$ and therefore $f$ admits an extension over $\beta X$, as required.

For a given complex $L$ we let $\mathcal{C}_{L}$ be the class of all metrizable compacta of extension dimension $\leq[L]$. We say that $X_{L}$ is a universal element for $\mathcal{C}_{L}$ if $X_{L}$ is a metrizable compactum with $e-\operatorname{dim} X_{L} \leq[L]$ which contains a topological copy of any metrizable compactum of extension dimension $\leq[L]$. The theorem below contains the main result of this note and follows directly from Theorem 3.4

Theorem 3.5. Let $L$ be a complex and let $\mathcal{C}_{L}$ be the class of all metrizable compacta of extension dimension $\leq[L]$. If $\mathcal{C}_{L}$ contains a universal element $X_{L}$ with the property $X_{L} \in A E([L], \mathcal{P})$, then $L$ is quasi-finite.

It follows from [8, Corollary 2.2] that none of the Eilenberg-Mac Lane complexes $K(G, n), n \geq 2$, and $G$ an Abelian group, is quasi-finite. Therefore Theorem 3.5 implies the following result.

Theorem 3.6. Let $G$ be a countably generated abelian group and let $n$ be an integer, $n \geq 2$. There is no universal compactum of given cohomological dimension $n$ with respect to the coefficient group $G$, which is an absolute extensor with respect to Polish spaces of cohomological dimension $\leq n$. 
In the case of integral cohomological dimension this theorem is similar to the observation made by Zarichnyi in [9].

\section{REFERENCES}

[1] N. Brodsky, A. Chigogidze, and A. Karasev, Approximations and selections of multivalued mappings of finite-dimensional spaces, JP Journal of Geometry and Topology 2, no. 1 (2002), 29-73. MR.1942626 (2003k:54013)

[2] A. Chigogidze, Cohomological dimension of Tychonov spaces, Topology Appl. 79, no. 3, (1997), 197-228. MR 1467214 (99f:55003)

[3] A. Chigogidze, Compactifications and universal spaces in extension theory, Proc. Am. Math. Soc. 128, no. 7 (2000), 2187-2190. MR.1653445 (2000m:55004)

[4] A. Chigogidze, Infinite dimensional topology and Shape theory, in: Handbook of Geometric Topology (eds.: R. Daverman, R. B. Sher), North-Holland, Amsterdam, 2002, 307-371. MR.1886673 (2003b:57030)

[5] A. N. Dranishnikov, The Eilenberg-Borsuk theorem for mappings in an arbitrary complex, Matem. Sb. 185 (1994), 81-90. MR.1272187 (95j:54028)

[6] A. N. Dranishnikov and J. Dydak, Extension dimension and extension types, Proc. Steklov Inst. Math. 212, no.1 (1996), 55-88. MR1635023 (99h:54049)

[7] A. Karasev, On two problems in extension theory, to appear in Topology Appl.

[8] A. Karasev and V. Valov, Extension dimension and quasi-finite $C W$ complexes, to appear in Topology Appl.

[9] M. Zarichnyi, Universal spaces and absolute extensors for integral cohomological dimension, Topology Appl., preprint.

Department of Computer Science and Mathematics, Nipissing University, 100 College Drive, P.O. Box 5002, North Bay, Ontario, Canada P1B 8L7

E-mail address: alexandk@nipissingu.ca

Department of Computer Science and Mathematics, Nipissing University, 100 College Drive, P.O. Box 5002, North Bay, Ontario, Canada P1B 8L7

E-mail address: veskov@nipissingu.ca 